\section{CONSEJO DE REDACCIÓN}

Neus Sanmartí - Directora DEPARTAMENT DE DID ÀCTICA DE LA MATEMÀTICA I DE LES CIÈNCIES EXPERIMENTALS, UNIVERSITAT AUTÒNOMA DE BARCELONA.

Bernardo Gómez - Director DEPARTAMENT DE DIDÀC TICA DE LES MATEMÃTIQUES, UNIVERSITAT DE VA. LENCIA.

Jaume Carrascosa PROFESOR DE EDUCACIÓN SECUNDARIA (VALENCIA)

Carles Furió DEPARTAMENT DE DIDACTICA DE LES CIENCIES EXPERIMENTALS DE LA UNIVERSITAT DE VALENCIA.

Susana García-Barros ASOCIACIÓN ESPAÑOLA DE PROFESORES E INVESTIGADORES DE DIDACTICA DE LAS CIENCIAS EXPERIMENTALES (APICE)

María José Gil Quílez DEPARTAMENTO DE DIDÁCTICA DE LAS CIENCIAS EXPERIMENTALES, UNIVERSIDAD DE ZARAGOZA

Julià Hinojosa PROFESOR DE EDUCACIÓN SECUNDARIA BARCELONA

Anna Marbà DEPARTAMENTO DE DIDÀCTICA DE LA MATEMATTICA I DE LES CIENCIES EXPERIMENTALS DE LAUNIVERSITAT AUTONOMA DE BARCELONA

Conxita Márquez DEPARTAMENT DE DIDACTICA DE LA MATEMÀTICA I DE LES CIENNCIES EXPERIMENTALS DE LA UNIVERSITAT AUTÒNOMA DE BARCELONA

Tomás Ortega DEPARTAMENTO DE DIDÁCTICA DE LA MATEMATICA DE LA UNIVERSIDAD DE VALLADOLTD Jordi Solbes (DEP. DE DIDÀTICA DE LES CIÈNCIES EXPERIMENTALS. UNIVERSITAT DE VALENCIA)

\section{CONSEJO ASESOR}

Carmen Azcárate DEPARTAMENT DE DIDÀCTICA DE LA MATEMÀTICA I DE LES CIENNCIES EXPERIMENTALS DE LA UNIVERSITAT AUTONOMA DE BARCELONA. Joan Bach (DEP. DE GEOLOGIA. UNIVERSITAT AUTONOMA DE BARCELONA) Enrique Banet (DEPARTAMENTO DE DIDÁCTICA DE LAS CIENCIAS DE LA UNIVERSIDAD DE MURCIA) António Cachapuz (DEP. DE DIDÁCTICA E TECNOLOGIA EDUCATIVA, UNIVERSIDADE DE AVEIRO, PORTUGAL) Juan Manuel Campanario (DEP. DE FÍSICA, UNIVERSIDAD DE ALCALÁ DE HENARES) Pedro Cañal (DEP. DE DIDÁCTICA DE LAS CIENCIAS, UNIVERSIDAD DE SEVILLA) Leonor Colombo (INSTITUTO DE FÍSICA, UNIVERSIDAD NACIONAL DE TUCUMÁN, ARGENTINA Moisés Coriat (DEP. DE DIDÁCTICA DE LA MATEMÁ TICAS, UNIVERSIDAD DE GRANADA) Mariona Espinet (DEPARTAMENT DE DIDÀCTICA DE LA MATEMÀTICA DE LES CIENNCIES EXPERIMENTALS DE LA UNIVERSITAT AUTONOMA DE BARCELONA) Eugenio Filloy (DEP. DE MATEMÁTICA EDUCATIVA, CENTRO DE INVESTIGACIÓN Y ESTUDIOS AVANZADOS DEL INSTITUTO POLITÉCNICO NACIONAL DE MÉXICO) Valentín Gavidia (DEPARTAMENT DE DIDÀCTICA DE LES CIENNCIES EXPERIMENTALS DE LA UNIVERSITAT DE VALENNCIA) André Giordan (LABORATOIRE DE DIDACTIQUE ET EPISTÉMOLOGIE DES SCIENCES, GINEBRA, SUIZA) Ricard Guerrero (DEP. DE MICROBIOLOGIA. UNIVERSITAT DE BAR CELONA) Jenaro Guisasola (DEP. DE FÍSICA APLICADA I. EUSKAL HERRIKO UNIBERTSITATEA-UNIVERSIDAD DEL PAÍS VASCO) Mercè Izquierdo DEPARTAMENT DE DIDÀCTICA DE LA MATEMATTICA I DE LES CIENCIES EXPERIMENTALS DE LA UNIVERSITAT AUTÒNOMA DE BARCELONA $\mathrm{M}^{\mathrm{a}}$ Pilar Jiménez-Aleixandre (DEP. DE DIDÁCTICA DE LAS CIENCIAS EXPERIMENTALES, UNIVERSIDAD DE SANTIAGO DE COMPOSTELA) José Lillo (DEP. DE DIDÁCTICA DE LAS CIENCIAS EXPERIMENTALES, UNIVERSIDAD DE VIGO) Salvador Llinares (DEPARTAMENT D'INNOVACIÓ I FORMACIÓ DIDÁCTICA, UNIVERSITAT D'ALACANT) Joan Antoni Llorens (DEP. DE QUÍMICA, UNIVERSITAT POLITËCNICA DE VALÈNCIA) Jean Louis Martinand (LIRES, ÉCOLE NORMALE SUPÉRIEURE DE CACHAN, FRANCIA)
Mercedes Martínez-Aznar (DEP. DE DIDÁCTICA DE LAS CIENCIAS EXPERIMENTALES, UNIVERSIDAD COMPLUTENSE DE MADRID) Joaquín Martínez Torregrosa (DEPARTAMENT DE DID ÀCTICA GENERAL I DE DIDÀC TIQUES ESPECÍFIQUES, UNIVERSITAT D'ALACANT) Isabel Martins (NÚCLEO DE TECNOLOGIA EDUCACIONAL PARA A SAÚDE, UNIVERSIDADE FEDERAL DO RIO DE JANEIRO, BRASIL) Vicente Mellado (DEP. DE DIDÁC TICA DE LAS CIENCIAS EXPERIMENTALES Y DE LAS MATEMÁTICAS, UNIVERSIDAD DE EXTREMADURA) Marco A. Moreira (INSTITUTO DE FÍSICA, UNIVERSIDADE FEDERAL DO RIO GRANDE DO SUD, BRASIL) Emilio Pedrinaci (IES DE GINES, SEVILLA) Francisco J. Perales (DEP. DE DIDÁCTICA DE LAS CIENCIAS EXPERIMENTALES, UNIVERSIDAD DE GRANADA) Rafael Porlán (DEP. DE DIDÁCTICA DE LAS CIENCIAS, UNIVERSIDAD DE SEVILLA) Antonio de Pro (DEP. DE DIDÁCTICA DE LAS CIENCIAS, UNIVERSIDAD DE MURCIA) Luis Puig (DEP. DE DIDÀCTICA DE LA MATEMÀTICA, UNIVERSITAT DE VALÈnCIA) Luis Rico (DEP. DE DIDÁCTICA DE LA MATEMÁTICA, UNIVERSIDAD DE GRANADA) Julia Salinas (DEP. DE FÍSICA DE LA FACULTAD DE CIENCIAS EXACTAS Y TECNOLOGÍA. UNIVERSIDAD NACIONAL DE TUCUMÁN, ARGENTINA) José Maria Sebastia (DEP. DE FÍSICA. UNIVERSIDAD SIMÓN BOLÍVAR, CARACAS, VENEZUELA) Laurence Viennot (LABORATOIRE DE DIDACTIQUE DE LA PHISIQUE DANS L'ENSEIGNEMENT SUPÉRIEUR, UNIVERSITÉ PARIS VII, FRANCIA) Paloma Varela(DEPARTAMENTO DE DIDÁCTICA DE LAS CIENCIAS EXPERIMENTALES. UNIVERSIDAD COMPLUTENSE DE MADRID)

La revista Enseñanza de las Ciencias está indexada en las bases de datos, directorios y plataformas de evaluación: SSCI (ISI)

Scopus DICE Cindoc (ISOC)

MATHDI

Latindex

Google Scholar

IRESIE

Compludoc

(C) Universitat de València, Universitat Autònoma de Barcelona y los autores, 2014

Edición impresa:

Publicacions de la Universitat de València i ICE de la Universitat Autònoma de Barcelona

Diseño de la cubierta, del interior y maquetación:

Celso Hernández de la Figuera y Gómez

Coordinación editorial:

Montserrat Andrés Díaz

Suscripciones:

http://revistes.uab.es/ensciencias/about/subscriptions

Recepción de orginales:

http://ensciencias.uab.es/about/

submissions\#onlineSubmissions

Dirección electrónica:

r.ensenanza.ciencias@uab.cat

ISSN (impreso): 0212-4521 y ISSN (digital): 2174-6486

Depósito legal: B-12373-1983

Impresión: GUADA IMPRESSORS, S.L. 